\title{
ON SOME INEQUALITIES OF CAUCHY-BUNYAKOVSKY-SCHWARZ TYPE AND APPLICATIONS
}

\author{
S. S. DRAGOMIR AND A. SOFO
}

Abstract. Some discrete inequalities of Cauchy-Bunyakovsky-Schwarz type for complex numbers with applications for the maximal deviation of a sequence from its weighted mean are given.

\section{Introduction}

The following result for complex numbers $a_{k}, b_{k}, k \in\{1, \ldots, n\}$ is well known in the literature as the Cauchy-Bunyakovsky-Schwarz (CBS) inequality:

$$
\left|\sum_{k=1}^{n} a_{k} b_{k}\right|^{2} \leq \sum_{k=1}^{n}\left|a_{k}\right|^{2} \sum_{k=1}^{n}\left|b_{k}\right|^{2}
$$

with equality if and only if there is a complex number $c \in \mathbb{C}$ such that $a_{k}=c \overline{b_{k}}$ for each $k \in$ $\{1, \ldots, n\}$, and $\overline{b_{k}}$ is the complex conjugate of $b_{k}$.

A simple proof of this statement can be achieved by utilising the following Lagrange identity for complex numbers (see [2, p. 3])

$$
\sum_{k=1}^{n}\left|a_{k}\right|^{2} \sum_{k=1}^{n}\left|b_{k}\right|^{2}-\left|\sum_{k=1}^{n} a_{k} b_{k}\right|^{2}=\frac{1}{2} \sum_{k, l=1}^{n}\left|\overline{a_{k}} b_{l}-\overline{a_{l}} b_{k}\right|^{2} .
$$

If $p_{k}, k \in\{1, \ldots, n\}$ are positive weights, then the weighted version of (1.1) can be stated as

$$
\left|\sum_{k=1}^{n} p_{k} a_{k} b_{k}\right|^{2} \leq \sum_{k=1}^{n} p_{k}\left|a_{k}\right|^{2} \sum_{k=1}^{n} p_{k}\left|b_{k}\right|^{2} .
$$

In [4], the following result connecting the unweighted version of the (CBS) inequality with the weighted one has been established (see also [2, p. 67-69]):

$$
\left(\sum_{k=1}^{n}\left|x_{k}\right|^{2} \sum_{k=1}^{n}\left|y_{k}\right|^{2}\right)^{\frac{1}{2}}-\left|\sum_{k=1}^{n} x_{k} y_{k}\right|
$$

Received December 25, 2006.

2000 Mathematics Subject Classification. 26D15, 26D10.

Key words and phrases. Cauchy-Bunyakovsky-Schwarz inequality, complex numbers, maximal deviation, probability density. 


$$
=\sup _{\mathbf{p} \in S_{n}(\mathbf{1})}\left\{\sum_{k=1}^{n} p_{k}\left|x_{k}\right|^{2} \sum_{k=1}^{n} p_{k}\left|y_{k}\right|^{2}-\left|\sum_{k=1}^{n} p_{k} x_{k} y_{k}\right|\right\},
$$

where $S_{n}(\mathbf{1})=\left\{\mathbf{p}=\left(p_{1}, \ldots, p_{n}\right) \mid 0 \leq p_{k} \leq 1\right.$ for each $\left.k \in\{1, \ldots, n\}\right\}$.

In the same paper the authors also established the following result concerning the length of summation in the CBS inequality:

$$
\begin{aligned}
& \left(\sum_{k=1}^{n} p_{k}\left|x_{k}\right|^{2} \sum_{k=1}^{n} p_{k}\left|y_{k}\right|^{2}\right)^{\frac{1}{2}}-\left|\sum_{k=1}^{n} p_{k} x_{k} y_{k}\right| \\
& \quad=\sup _{I \subseteq\{1, \ldots, n\}}\left[\left(\sum_{k \in I} p_{k}\left|x_{k}\right|^{2} \sum_{k \in I} p_{k}\left|y_{k}\right|^{2}\right)^{\frac{1}{2}}-\left|\sum_{k=1}^{n} p_{k} x_{k} y_{k}\right|\right]
\end{aligned}
$$

and

$$
\begin{aligned}
& \left(\sum_{k=1}^{n} p_{k}\left|x_{k}\right|^{2} \sum_{k=1}^{n} p_{k}\left|y_{k}\right|^{2}\right)^{\frac{1}{2}}-\left|\sum_{k=1}^{n} p_{k} x_{k} y_{k}\right| \\
& \quad \geq \max _{1 \leq k<l \leq n}\left\{\left[p_{k}\left|x_{k}\right|^{2}+p_{l}\left|x_{l}\right|^{2}\right]^{\frac{1}{2}}\left[p_{k}\left|y_{k}\right|^{2}+p_{l}\left|y_{l}\right|^{2}\right]^{\frac{1}{2}}-\left|p_{k} x_{k} y_{k}+p_{l} x_{l} y_{l}\right|\right\},
\end{aligned}
$$

for any $x_{k}, y_{k} \in \mathbb{C}, k \in\{1, \ldots, n\}$.

For some historical facts on CBS inequality, see [9] and [2]. Refinements of this inequality are provided in [1], [6], [8] and in the Chapter 2 of [2]. Other results related to CBS inequality may be found in [5] and [7].

The aim of the present paper is to establish some inequalities of CBS type under the supplementary assumption that either $\sum_{k=1}^{n} x_{k} y_{k}=0$ or $\sum_{k=1}^{n} p_{k} x_{k} y_{k}=0$, when the weighted version is considered. Applications that provide upper bounds for the maximal deviation of a sequence $x_{k}$ from the weighted mean $\sum_{j=1}^{n} p_{j} x_{j}$, namely, for the quantity

$$
\max _{k \in\{1, \ldots, n\}}\left|x_{k}-\sum_{j=1}^{n} p_{j} x_{j}\right|
$$

where $x_{k} \in \mathbb{C}, p_{k} \geq 0, k \in\{1, \ldots, n\}, \sum_{k=1}^{n} p_{k}=1$, are also given.

\section{The Results}

The following result holds:

Theorem 1. Let $a_{k}, b_{k} \in \mathbb{C}, k \in\{1, \ldots, n\}, n \geq 2$ with the property that

$$
\sum_{k=1}^{n} a_{k} b_{k}=0
$$

Then

$$
\max _{i \in\{1, \ldots, n\}}\left\{\left|a_{i} b_{i}\right|\right\} \leq \frac{1}{2}\left(\sum_{k=1}^{n}\left|a_{k}\right|^{2}\right)^{\frac{1}{2}}\left(\sum_{k=1}^{n}\left|b_{k}\right|^{2}\right)^{\frac{1}{2}} .
$$


The constant $\frac{1}{2}$ in (2.2) is best possible in the sense that it cannot be replaced by a smaller constant.

Proof. For any $i \in\{1, \ldots, n\}$, we have

$$
a_{i} b_{i}=-\sum_{\substack{k=1 \\ k \neq i}}^{n} a_{k} b_{k}
$$

Taking the modulus in (2.3) we have

$$
\begin{aligned}
\left|a_{i} b_{i}\right| & =\left|\sum_{\substack{k=1 \\
k \neq i}}^{n} a_{k} b_{k}\right| \leq\left(\sum_{\substack{k=1 \\
k \neq i}}^{n}\left|a_{k}\right|^{2}\right)^{\frac{1}{2}}\left(\sum_{\substack{k=1 \\
k \neq i}}^{n}\left|b_{k}\right|^{2}\right)^{\frac{1}{2}} \\
& =\left(\sum_{k=1}^{n}\left|a_{k}\right|^{2}-\left|a_{i}\right|^{2}\right)^{\frac{1}{2}}\left(\sum_{k=1}^{n}\left|b_{k}\right|^{2}-\left|b_{i}\right|^{2}\right)^{\frac{1}{2}},
\end{aligned}
$$

for any $i \in\{1, \ldots, n\}$, where we used the Cauchy-Bunyakovsky-Schwarz inequality to state the required inequality in (2.4).

Utilising the elementary inequality for real numbers

$$
\left(\alpha^{2}-\beta^{2}\right)^{\frac{1}{2}}\left(\gamma^{2}-\delta^{2}\right)^{\frac{1}{2}} \leq \alpha \gamma-\beta \delta,
$$

provided $\alpha, \beta, \gamma, \delta>0$ and $\alpha \geq \beta, \gamma \geq \delta$, we have

$$
\begin{aligned}
& \left(\sum_{k=1}^{n}\left|a_{k}\right|^{2}-\left|a_{i}\right|^{2}\right)^{\frac{1}{2}}\left(\sum_{k=1}^{n}\left|b_{k}\right|^{2}-\left|b_{i}\right|^{2}\right)^{\frac{1}{2}} \\
& \quad=\left\{\left[\left(\sum_{k=1}^{n}\left|a_{k}\right|^{2}\right)^{\frac{1}{2}}\right]^{2}-\left|a_{i}\right|^{2}\right\}^{\frac{1}{2}}\left\{\left[\left(\sum_{k=1}^{n}\left|b_{k}\right|^{2}\right)^{\frac{1}{2}}\right]^{2}-\left|b_{i}\right|^{2}\right\}^{\frac{1}{2}} \\
& \quad \leq\left(\sum_{k=1}^{n}\left|a_{k}\right|^{2}\right)^{\frac{1}{2}}\left(\sum_{k=1}^{n}\left|b_{k}\right|^{2}\right)^{\frac{1}{2}}-\left|a_{i} b_{i}\right|,
\end{aligned}
$$

for each $i \in\{1, \ldots, n\}$.

Now, on making use of (2.4) and (2.5) we get the desired inequality (2.2).

To prove the sharpness of the constant, we assume that the inequality (2.2) holds true for a constant $C>0$, i.e.,

$$
\max _{i \in\{1, \ldots, n\}}\left|a_{i} b_{i}\right| \leq C\left(\sum_{k=1}^{n}\left|a_{k}\right|^{2}\right)^{\frac{1}{2}}\left(\sum_{k=1}^{n}\left|b_{k}\right|^{2}\right)^{\frac{1}{2}},
$$

provided $a_{k}, b_{k}, k \in\{1, \ldots, n\}(n \geq 2)$ are complex numbers such that $\sum_{k=1}^{n} a_{k} b_{k}=0$.

Now, for $n=2$, choose $a_{1}=a, a_{2}=-b, b_{1}=b, b_{2}=-a$ with $a, b>0$. Then $a_{1} b_{1}+a_{2} b_{2}=0$, $\left|a_{1} b_{1}\right|=\left|a_{2} b_{2}\right|=a b$ and by (2.6) we get

$$
a b \leq C\left(a^{2}+b^{2}\right) \text { for } a, b>0 .
$$


Choosing in (2.7) $a=b=1$, we deduce $C \geq \frac{1}{2}$ and the proof is complete.

The following corollary is of interest.

Corollary 1. Let $x_{k} \in \mathbb{C}, k \in\{1, \ldots, n\}$ and $p_{k}, k \in\{1, \ldots, n\}$ be a probability sequence, i.e., $p_{k} \geq 0, k \in\{1, \ldots, n\}$ and $\sum_{k=1}^{n} p_{k}=1$. Then we have the inequality:

$$
\begin{aligned}
\max _{i \in\{1, \ldots, n\}}\left\{p_{i}\left|x_{i}-\sum_{j=1}^{n} p_{j} x_{j}\right|\right\} \\
\quad \leq \frac{1}{2}\left(\sum_{k=1}^{n} p_{k}^{2}\right)^{\frac{1}{2}}\left(\sum_{k=1}^{n}\left|x_{k}-\sum_{j=1}^{n} p_{j} x_{j}\right|^{2}\right)^{\frac{1}{2}} \\
\quad=\frac{1}{2}\left(\sum_{k=1}^{n} p_{k}^{2}\right)^{\frac{1}{2}}\left\{\sum_{k=1}^{n}\left|x_{k}\right|^{2}+n\left|\sum_{j=1}^{n} p_{j} x_{j}\right|^{2}-2 \operatorname{Re}\left[\left(\sum_{k=1}^{n} x_{k}\right)\left(\sum_{j=1}^{n} p_{j} \bar{x}_{j}\right)\right]\right\}^{\frac{1}{2}} .
\end{aligned}
$$

Proof. If we choose $a_{k}=p_{k}, b_{k}:=x_{k}-\sum_{j=1}^{n} p_{j} x_{j}$, then

$$
\sum_{k=1}^{n} a_{k} b_{k}=\sum_{k=1}^{n} p_{k}\left(x_{k}-\sum_{j=1}^{n} p_{j} x_{j}\right)=0
$$

and the condition (2.1) is satisfied.

Applying the inequality (2.2), we obtain

$$
\begin{aligned}
\max _{i \in\{1, \ldots, n\}}\left\{p_{i}\left|x_{i}-\sum_{j=1}^{n} p_{j} x_{j}\right|\right\} \\
\leq \frac{1}{2}\left(\sum_{k=1}^{n} p_{k}^{2}\right)^{\frac{1}{2}}\left(\sum_{k=1}^{n}\left|x_{k}-\sum_{j=1}^{n} p_{j} x_{j}\right|^{2}\right)^{\frac{1}{2}} \\
=\frac{1}{2}\left(\sum_{k=1}^{n} p_{k}^{2}\right)^{\frac{1}{2}}\left(\sum_{k=1}^{n}\left|x_{k}\right|^{2}-2 \operatorname{Re}\left(\sum_{k=1}^{n} x_{k} \cdot \sum_{j=1}^{n} p_{j} \overline{x_{j}}\right)+n\left|\sum_{j=1}^{n} p_{j} x_{j}\right|^{2}\right)^{\frac{1}{2}}
\end{aligned}
$$

and the inequality (2.8) is obtained.

Remark 1. If $\min _{i \in\{1, \ldots, n\}} p_{i}=p_{m}>0$, then from (2.8) we can obtain a coarser and perhaps more useful inequality, providing some upper bounds for the maximal deviation of $x_{k}$ from the weighted mean $\sum_{j=1}^{n} p_{j} x_{j}$, namely,

$$
\max _{k \in\{1, \ldots, n\}}\left|x_{k}-\sum_{j=1}^{n} p_{j} x_{j}\right| \leq \frac{1}{2 p_{m}}\left(\sum_{k=1}^{n} p_{k}^{2}\right)^{\frac{1}{2}}\left(\sum_{k=1}^{n}\left|x_{k}-\sum_{j=1}^{n} p_{j} x_{j}\right|^{2}\right)^{\frac{1}{2}}
$$

The following weighted version of Theorem 1 may be stated as well: 
Theorem 2. Let $x_{k}, y_{k} \in \mathbb{C}, k \in\{1, \ldots, n\}$ and $p_{k}, k \in\{1, \ldots, n\}$ be a probability sequence with the property that

$$
\sum_{k=1}^{n} p_{k} x_{k} y_{k}=0
$$

Then

$$
\max _{i \in\{1, \ldots, n\}}\left\{p_{i}\left|x_{i} y_{i}\right|\right\} \leq \frac{1}{2}\left(\sum_{k=1}^{n} p_{k}\left|x_{k}\right|^{2}\right)^{\frac{1}{2}}\left(\sum_{k=1}^{n} p_{k}\left|y_{k}\right|^{2}\right)^{\frac{1}{2}} .
$$

The constant $\frac{1}{2}$ in (2.11) is best possible in (2.11).

Proof. It follows from Theorem 1 on choosing $a_{k}=\sqrt{p_{k}} x_{k}, b_{k}=\sqrt{p_{k}} y_{k}$.

Remark 2. One should notice that Theorem 1 and Theorem 2 are equivalent in the sense that one implies the other.

The above result provides the opportunity to obtain a different bound for the maximal deviation of $x_{k}$ from the weighted mean.

Corollary 2. With the assumptions in Corollary 1, we have the inequality:

$$
\begin{aligned}
\max _{i \in\{1, \ldots, n\}}\left\{p_{i}\left|x_{i}-\sum_{j=1}^{n} p_{j} x_{j}\right|\right\} & \leq \frac{1}{2}\left(\sum_{k=1}^{n} p_{k}\left|x_{k}-\sum_{j=1}^{n} p_{j} x_{j}\right|^{2}\right)^{\frac{1}{2}} \\
& =\frac{1}{2}\left[\sum_{k=1}^{n} p_{k}\left|x_{k}\right|^{2}-\left|\sum_{j=1}^{n} p_{j} x_{j}\right|^{2}\right]^{\frac{1}{2}} .
\end{aligned}
$$

Proof. Follows by Theorem 2 on choosing $y_{k}=1, k \in\{1, \ldots, n\}$.

Remark 3. If $\min _{i \in\{1, \ldots, n\}} p_{i}=p_{m}>0$, then

$$
\max _{i \in\{1, \ldots, n\}}\left|x_{k}-\sum_{j=1}^{n} p_{j} x_{j}\right| \leq \frac{1}{2 p_{m}}\left(\sum_{k=1}^{n} p_{k}\left|x_{k}-\sum_{j=1}^{n} p_{j} x_{j}\right|^{2}\right)^{\frac{1}{2}} .
$$

Remark 4. It is natural to ask which of the bounds for the maximal deviation

$$
\max _{i \in\{1, \ldots, n\}}\left\{p_{i}\left|x_{i}-\sum_{j=1}^{n} p_{j} x_{j}\right|\right\}
$$

provided by (2.8) and (2.12) are better and when, respectively?

For $n=2$, let $p_{1}=p, p_{2}=1-p, p \in[0,1], x_{1}=x, x_{2}=y$, then we have the specific case of

$$
\begin{aligned}
B_{1}(p, x, y) & :=\frac{1}{2}\left[p^{2}+(1-p)^{2}\right]^{\frac{1}{2}}\left[(x-p x-(1-p) y)^{2}+(y-p x-(1-p) y)^{2}\right]^{\frac{1}{2}} \\
& =\frac{1}{2}\left[p^{2}+(1-p)^{2}\right]^{\frac{1}{2}}\left[(1-p)^{2}(x-y)^{2}+p^{2}(x-y)^{2}\right]^{\frac{1}{2}}
\end{aligned}
$$




$$
=\frac{1}{2} \cdot\left[p^{2}+(1-p)^{2}\right]|x-y|
$$

and

$$
\begin{aligned}
B_{2}(p, x, y & :=\frac{1}{2}\left[p(x-p x-(1-p) y)^{2}+(1-p)(y-p x-(1-p) y)^{2}\right]^{\frac{1}{2}} \\
& =\frac{1}{2}\left[p(1-p)^{2}(x-y)^{2}+(1-p) p^{2}(x-y)^{2}\right]^{\frac{1}{2}} \\
& =\frac{1}{2} \cdot \sqrt{p(1-p)}|x-y| .
\end{aligned}
$$

Since $p^{2}+(1-p)^{2} \geq \sqrt{p(1-p)}$ for $p \in[0,1]$, we have that the bound (2.12) is always better than (2.8) for $n=2$.

Remark 5. For $n=3, p_{1}=p, p_{2}=q, p_{3}=r, x_{1}=x, x_{2}=y, x_{3}=z$, we should compare the bounds

$$
\begin{aligned}
B_{1}(p, q, r, x, y, z)= & \frac{1}{2}\left(p^{2}+q^{2}+r^{2}\right)^{\frac{1}{2}} \times\left[p(x-p x-q y-r z)^{2}\right. \\
& \left.+q(y-p x-q y-r z)^{2}+r(z-p x-q y-r z)^{2}\right]^{\frac{1}{2}}
\end{aligned}
$$

and

$$
\begin{aligned}
B_{2}(p, q, r, x, y, z)= & \frac{1}{2}\left[p(x-p x-q y-r z)^{2}+q(y-p x-q y-r z)^{2}\right. \\
& \left.+r(z-p x-q y-r z)^{2}\right]^{\frac{1}{2}} .
\end{aligned}
$$

The plot of the function

$$
\Delta(0.1,0.5,0.4, x, y,-4)=B_{1}(0.1,0.5,0.4, x, y,-4)-B_{2}(0.1,0.5,0.4, x, y,-4)
$$

on the box $[0,6] \times[8,10]$ shows that one bound is not always better the other (see Figure 1):

Remark 6. In the case of uniform distribution, i.e., when $p_{i}=\frac{1}{n}, i \in\{1, \ldots, n\}$, we obtain from both inequalities (2.8) and (2.12) the same result:

$$
\begin{aligned}
\max _{k \in\{1, \ldots, n\}}\left|x_{k}-\frac{1}{n} \sum_{j=1}^{n} x_{j}\right| & \leq \frac{1}{2} \sqrt{n} \sum_{k=1}^{n}\left|x_{k}-\frac{1}{n} \sum_{j=1}^{n} x_{j}\right|^{2} \\
& =\frac{1}{2}\left[n \sum_{k=1}^{n}\left|x_{k}\right|^{2}-\left|\sum_{k=1}^{n} x_{k}\right|^{2}\right]^{\frac{1}{2}} .
\end{aligned}
$$

\section{Related Results}

The following result may be stated as well. 


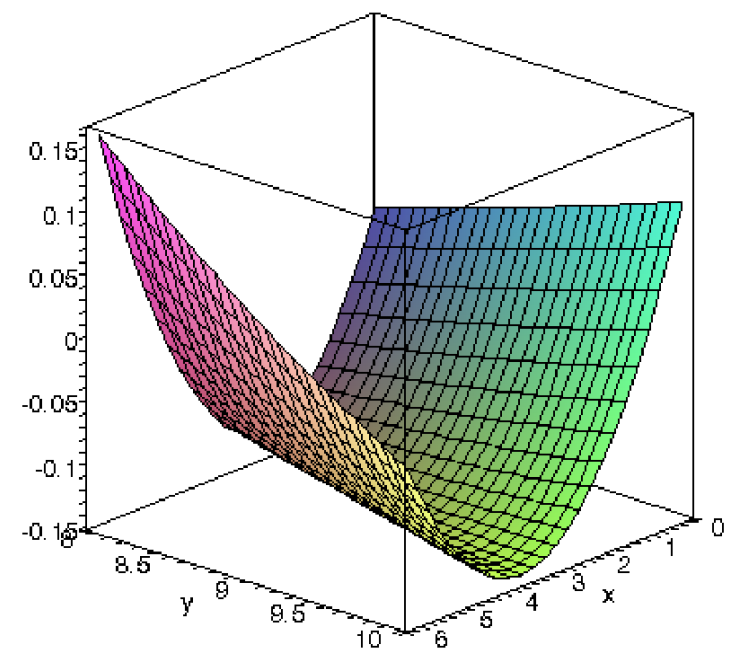

Figure 1. Plot of the difference $\Delta(0.1,0.5,0.4, x, y,-4)$ showing a transition from positive to negative.

Theorem 3. Let $a_{k}, b_{k} \in \mathbb{C} \backslash\{0\}, k \in\{1, \ldots, n\}$ so that $\sum_{k=1}^{n} a_{k} b_{k}=0$. Then for any probability sequence $p_{k}, k \in\{1, \ldots, n\}$, we have:

$$
\frac{\sum_{j=1}^{n} p_{j}\left|a_{j}\right|^{2}}{\sum_{k=1}^{n}\left|a_{k}\right|^{2}}+\frac{\sum_{j=1}^{n} p_{j}\left|b_{j}\right|^{2}}{\sum_{k=1}^{n}\left|b_{k}\right|^{2}} \leq 1
$$

Proof. We know, from the proof of Theorem 1, that

$$
\begin{aligned}
\left|a_{i} b_{i}\right|^{2} & \leq\left(\sum_{k=1}^{n}\left|a_{k}\right|^{2}-\left|a_{i}\right|^{2}\right)\left(\sum_{k=1}^{n}\left|b_{k}\right|^{2}-\left|b_{i}\right|^{2}\right) \\
& =\sum_{k=1}^{n}\left|a_{k}\right|^{2} \sum_{k=1}^{n}\left|b_{k}\right|^{2}+\left|a_{i}\right|^{2}\left|b_{i}\right|^{2}-\left|a_{i}\right|^{2} \sum_{k=1}^{n}\left|b_{k}\right|^{2}-\left|b_{i}\right|^{2} \sum_{k=1}^{n}\left|a_{k}\right|^{2}
\end{aligned}
$$

which is clearly equivalent with

$$
\left|a_{i}\right|^{2} \sum_{k=1}^{n}\left|b_{k}\right|^{2}+\left|b_{i}\right|^{2} \sum_{k=1}^{n}\left|a_{k}\right|^{2} \leq \sum_{k=1}^{n}\left|a_{k}\right|^{2} \sum_{k=1}^{n}\left|b_{k}\right|^{2}
$$

for each $i \in\{1, \ldots, n\}$.

Now, if we multiply (3.2) by $p_{i} \geq 0$ and sum over $i \in\{1, \ldots, n\}$, we deduce:

$$
\sum_{i=1}^{n} p_{i}\left|a_{i}\right|^{2} \sum_{k=1}^{n}\left|b_{k}\right|^{2}+\sum_{i=1}^{n} p_{i}\left|b_{i}\right|^{2} \sum_{k=1}^{n}\left|a_{k}\right|^{2} \leq \sum_{k=1}^{n}\left|a_{k}\right|^{2} \sum_{k=1}^{n}\left|b_{k}\right|^{2}
$$


which is clearly equivalent with (3.1).

Corollary 3. With the assumptions of the above theorem, we have:

$$
\sum_{i=1}^{n} p_{i}\left|a_{i}\right|^{2} \sum_{i=1}^{n} p_{i}\left|b_{i}\right|^{2} \leq \frac{1}{4} \sum_{k=1}^{n}\left|a_{k}\right|^{2} \sum_{k=1}^{n}\left|b_{k}\right|^{2} .
$$

The constant $\frac{1}{4}$ is best possible in (3.4).

Proof. On utilising the inequality $\alpha^{2}+\beta^{2} \geq 2 \alpha \beta, \alpha, \beta \in \mathbb{R}_{+}$, we have

$$
\begin{aligned}
& \sum_{j=1}^{n} p_{j}\left|a_{j}\right|^{2} \sum_{k=1}^{n}\left|b_{k}\right|^{2}+\sum_{j=1}^{n} p_{j}\left|b_{j}\right|^{2} \sum_{k=1}^{n}\left|a_{k}\right|^{2} \\
& \quad \geq 2\left(\sum_{j=1}^{n} p_{j}\left|a_{j}\right|^{2} \sum_{j=1}^{n} p_{j}\left|b_{j}\right|^{2}\right)^{\frac{1}{2}}\left(\sum_{k=1}^{n}\left|a_{k}\right|^{2} \sum_{k=1}^{n}\left|b_{k}\right|^{2}\right)^{\frac{1}{2}} .
\end{aligned}
$$

Now, by (3.3) and (3.5) we deduce the desired inequality (3.4).

To prove the sharpness of the constant, we assume that (3.4) holds true with a $D>0$, i.e.,

$$
\sum_{j=1}^{n} p_{j}\left|a_{j}\right|^{2} \sum_{j=1}^{n} p_{j}\left|b_{j}\right|^{2} \leq D \sum_{k=1}^{n}\left|a_{k}\right|^{2} \sum_{k=1}^{n}\left|b_{k}\right|^{2},
$$

provided $\sum_{k=1}^{n} a_{k} b_{k}=0, n \geq 2$.

For $n=2$, we choose $a_{1}=a, a_{2}=-b, b_{1}=b, b_{2}=-a$ and $p_{1}=p, p_{2}=1-p$ to get:

$$
\left[p a^{2}+(1-p) b^{2}\right]\left[p b^{2}+(1-p) a^{2}\right] \leq D\left[a^{2}+b^{2}\right]^{2} \text {. }
$$

If in (3.6) we choose $p=\frac{1}{2}$, then we get

$$
\frac{1}{4}\left(a^{2}+b^{2}\right)^{2} \leq D\left(a^{2}+b^{2}\right)^{2},
$$

which shows that $D \geq \frac{1}{4}$.

Corollary 4. Let $x_{k} \in \mathbb{C}, k \in\{1, \ldots, n\}$ and $p_{k}, k \in\{1, \ldots, n\}$ be a probability sequence. Then:

$$
\begin{aligned}
\sum_{k=1}^{n} p_{k}\left|x_{k}\right|^{2}-\left|\sum_{k=1}^{n} p_{k} x_{k}\right|^{2} & =\sum_{j=1}^{n} p_{j}\left|x_{j}-\sum_{l=1}^{n} p_{l} x_{l}\right|^{2} \\
& \leq \frac{1}{4} \cdot \frac{\sum_{k=1}^{n} p_{k}^{2}}{\sum_{k=1}^{n} p_{k}^{3}} \sum_{k=1}^{n}\left|x_{k}-\sum_{l=1}^{n} p_{l} x_{l}\right|^{2} .
\end{aligned}
$$

Proof. It is obvious by (3.4) on choosing $a_{k}=p_{k}$ and $b_{k}=x_{k}-\sum_{l=1}^{n} p_{l} x_{l}, k \in\{1, \ldots, n\}$.

The following result that provides a refinement of Theorem 2 should be noted. 
Theorem 4. Let $x_{k}, y_{k} \in \mathbb{C}, k \in\{1, \ldots, n\}$ and $p_{k}, k \in\{1, \ldots, n\}$ be a probability sequence with the property that

$$
\sum_{k=1}^{n} p_{k} x_{k} y_{k}=0
$$

Then

$$
\begin{aligned}
& \max _{i \in\{1, \ldots, n\}}\left\{p_{i}\left|x_{i} y_{i}\right|\right\} \\
& \leq \frac{1}{2} \cdot \frac{\max _{i \in\{1, \ldots, n\}}\left[p_{i}\left|x_{i}\right|^{2} \sum_{k=1}^{n} p_{k}\left|y_{k}\right|^{2}+p_{i}\left|y_{i}\right|^{2} \sum_{k=1}^{n} p_{k}\left|x_{k}\right|^{2}\right]}{\left(\sum_{k=1}^{n} p_{k}\left|x_{k}\right|^{2} \sum_{k=1}^{n} p_{k}\left|y_{k}\right|^{2}\right)^{\frac{1}{2}}} \\
& \quad \leq \frac{1}{2} \cdot\left(\sum_{k=1}^{n} p_{k}\left|x_{k}\right|^{2} \sum_{k=1}^{n} p_{k}\left|y_{k}\right|^{2}\right)^{\frac{1}{2}} \cdot
\end{aligned}
$$

Proof. As in the proof of Theorem 1, we have

$$
p_{i}\left|x_{i} y_{i}\right| \leq\left(\sum_{k=1}^{n} p_{k}\left|x_{k}\right|^{2}-p_{i}\left|x_{i}\right|^{2}\right)^{\frac{1}{2}}\left(\sum_{k=1}^{n} p_{k}\left|y_{k}\right|^{2}-p_{i}\left|y_{i}\right|^{2}\right)^{\frac{1}{2}},
$$

which gives

$$
\begin{aligned}
p_{i}^{2}\left|x_{i} y_{i}\right|^{2} & \leq\left(\sum_{k=1}^{n} p_{k}\left|x_{k}\right|^{2}-p_{i}\left|x_{i}\right|^{2}\right)\left(\sum_{k=1}^{n} p_{k}\left|y_{k}\right|^{2}-p_{i}\left|y_{i}\right|^{2}\right) \\
& =\sum_{k=1}^{n} p_{k}\left|x_{k}\right|^{2} \sum_{k=1}^{n} p_{k}\left|y_{k}\right|^{2}+p_{i}^{2}\left|x_{i}\right|^{2}\left|y_{i}\right|^{2}-p_{i}\left|x_{i}\right|^{2} \sum_{k=1}^{n} p_{k}\left|y_{k}\right|^{2}-p_{i}\left|y_{i}\right|^{2} \sum_{k=1}^{n} p_{k}\left|x_{k}\right|^{2},
\end{aligned}
$$

i.e.,

$$
p_{i}\left|x_{i}\right|^{2} \sum_{k=1}^{n} p_{k}\left|y_{k}\right|^{2}+p_{i}\left|y_{i}\right|^{2} \sum_{k=1}^{n} p_{k}\left|x_{k}\right|^{2} \leq \sum_{k=1}^{n} p_{k}\left|x_{k}\right|^{2} \sum_{k=1}^{n} p_{k}\left|y_{k}\right|^{2}
$$

for each $i \in\{1, \ldots, n\}$.

Taking the maximum in (3.9) over $i \in\{1, \ldots, n\}$, we get the second inequality in (3.8).

The first inequality follows by the elementary fact that

$$
\begin{aligned}
p_{i}\left|x_{i}\right|^{2} \sum_{k=1}^{n} p_{k}\left|y_{k}\right|^{2}+p_{i}\left|y_{i}\right|^{2} \sum_{k=1}^{n} p_{k}\left|x_{k}\right|^{2} & \\
& \geq 2 p_{i}\left|x_{i}\right|\left|y_{i}\right|\left(\sum_{k=1}^{n} p_{k}\left|x_{k}\right|^{2}\right)^{\frac{1}{2}}\left(\sum_{k=1}^{n} p_{k}\left|y_{k}\right|^{2}\right)^{\frac{1}{2}}
\end{aligned}
$$

for each $i \in\{1, \ldots, n\}$.

Remark 7. The inequality (3.8) is obviously a refinement of the inequality (2.11) in Theorem 2. However, the inequality (3.8) is not apparently useful in deriving upper bounds for the maximal deviation of $x_{k}$ from its weighted mean $\sum_{j=1}^{n} p_{j} x_{j}$, as the inequality (2.11). 


\section{References}

[1] N. G. de Bruijn, Problem 12, Wisk. Opgaven, 21(1960), 12-14.

[2] S. S. Dragomir, Discrete Inequalities of the Cauchy-Bunykovsky-Schwarz Type, Nova Science Publishers Inc., N.Y., 2004.

[3] S. S. Dragomir, Reverses of the Cauchy-Bunyakovsky-Schwarz inequality for n-tuples of complex numbers, Bull. Austral. Math. Soc. 69(2004), 465-480.

[4] S. S. Dragomir and B. Mond, On the superadditivity and monotonicity of Schwarz's inequality in inner product spaces, Contributions Ser. Math. Sci. Macedonian Acad. Sci. 15(1994), 5-22.

[5] S. S. Dragomir and B. Mond, Some mappings associated with Cauchy-Buniakowski-Schwarz's inequality in inner product spaces. Soochow J. Math. 21(1995), 413-426.

[6] J. W. Hovenier, Sharpening Cauchy's inequality, J. Math. Anal. Appl. 186(1994), 156-160.

[7] Y.-C. Li, S.-Y. Shaw, A proof of Hölder's inequality using the Cauchy-Schwarz inequality, J. Inequal. Pure Appl. Math. 7(2006), Article 62, pp.3.

[8] Z. Liu, Remark on a refinement of the Cauchy-Schwarz inequality, J. Math. Anal. Appl. 218(1998), 13-21.

[9] P. Schreiber, The Cauchy-Bunyakovsky-Schwarz inequality, Hermann Graßmann (Lieschow, 1994), 64-70, Ernst-Moritz-Arndt Univ., Greifswald, 1995.

Research Group in Mathematical Inequalities \& Applications, School of Engineering \& Science, Victoria University, PO Box 14428, Melbourne City, 8001, VIC, Australia.

E-mail: sever.dragomir@vu.edu.au

Research Group in Mathematical Inequalities \& Applications, School of Engineering \& Science, Victoria University, PO Box 14428, Melbourne City, 8001, VIC, Australia.

E-mail: anthony.sofo@vu.edu.au 